director of the Australian Research Council Centre of Excellence for Translational Photosynthesis in Acton. "We have to look at radical solutions." Reducing food loss and waste (see page S6), and making more-sustainable dietary choices (see page S18) will help. But increasing the potential yield of crops to produce more food without using extra land, water or fertilizer would be a powerful boost to ensuring people have access to enough food. The hope is that with genetic modification of photosynthesis we will enter a new era of yield improvements.

\section{CATCHING THE SUN}

Photosynthesis is the chain of metabolic reactions through which plants convert energy from the Sun, $\mathrm{CO}_{2}$ and water into the molecules they need. The process converts only about $5 \%$ of the energy that plants receive into biomass. "You would think a billion years of evolution would have optimized photosynthesis by now," says Niyogi, but things aren't so simple. For one thing, cultivated crops face different limiting factors to wild plants. "In nature, plants are usually limited by drought stress, nutrients and pathogens,"

he says. But modern farming methods and tools such as irrigation, fertilizer and pesticides have lessened these challenges, affording cultivated crops the luxury of bumping up against the inefficiencies of light absorption.

Niyogi's research centres on a fundamental process called photoprotection. High levels of sunlight can damage plant cells, so to protect themselves they make pigments that temporarily deflect excess energy when sunlight is too bright - a botanical sunscreen. When the light levels shift, perhaps because of the movement of leaves that shade others or a change in cloud cover, plant cells ramp photosynthesis back up - but it takes a few minutes. In densely planted crops, where a canopy of leaves creates dynamic patterns of light and shade, a lot of productive time is lost to this photoprotection lag.

Niyogi's research focuses on a photoprotection mechanism called non-photochemical quenching (NPQ), which reduces yield by an estimated $20 \%$. His group has had promising results from the upregulation of three genes in tobacco involved in two forms of NPQ that include the protective pigment zeaxanthin. But positive results in the lab are no guarantee of success in a field crop. It can be difficult to get plants to stably express new genes over multiple generations. And of course, field conditions can vary widely from the controlled environment inside growth cabinets.

Niyogi's team is part of a multi-institution project - called Realizing Increased Photosynthetic Efficiency (RIPE) - to increase the yield of food crops in developing countries through photosynthesis. As part of the programme, the Berkeley researchers worked with plant biologist Stephen Long, who directs the wider RIPE project, and his team at the University of Illinois at Urbana-Champaign to do field tests of the engineered light-handling mechanism in tobacco. Tobacco is a good model for studying photoprotection because it has a dense canopy of leaves, like maize.

RIPE's computer models, which simulate the reactions of photosynthesis, predicted a $20 \%$ improvement in yield for tobacco plants with genetically altered NPQ pathways. That's a big change - an improvement of just a few per cent achieved without upping the pressure on land, water or fertilizer would make a significant difference to farmers. Because this kind of research can be difficult to translate to field trials, when the researchers measured the dry weight, and saw the tobacco yield had been boosted by $15 \%{ }^{1}$, they were delighted. "Yes! We got more!" Long says.

They are now planning to test whether the photoprotection boost works in cowpea (Vigna unguiculata), a major source of protein in subSaharan Africa, in partnership with researchers

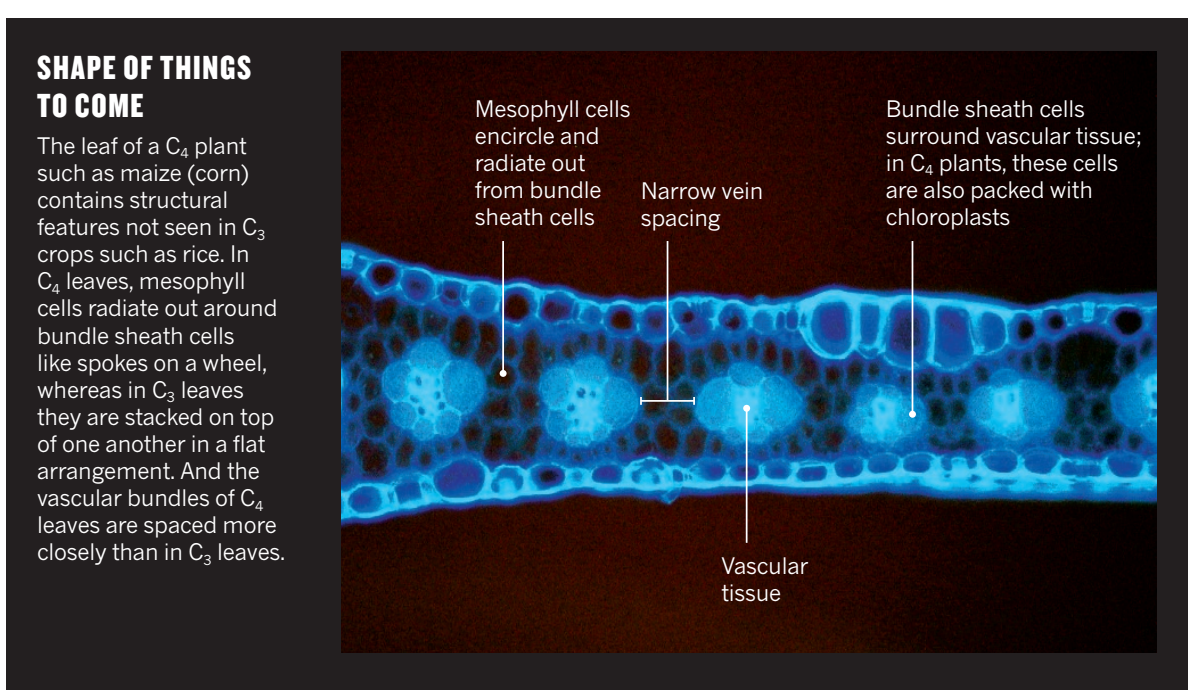

at the Commonwealth Scientific and Industrial Research Organisation in Australia and cowpea breeders in West Africa. And in Illinois, the researchers are translating the work to rice.

Tinkering with photoprotection is not the only way to improve plants' use of light energy. Anastasios Melis, a plant biologist at the University of California, Berkeley, is working on reducing the amount of the pigment chlorophyll inside chloroplasts, plants' photosynthetic organelles. Melis knows that this sounds counter-intuitive. But consider the way in which a canopy of densely planted crops shades many of the leaves below, he says. More-transparent leaves with less chlorophyll will allow more light to pass through to lower leaves - improving the productivity of the plant as a whole.

Researchers are also studying the infraredabsorbing chlorophylls made by some types of photosynthetic cyanobacteria ${ }^{2}$. Infrared radiation is normally considered to be outside the range that can be used by plants for photosynthesis. If scientists can map out the details of how bacterial chlorophylls are produced, and integrate them into crops, the number of available photons could increase by about 19\%, allowing the modified plants to capture more solar energy. "We cannot change the intensity of the sunlight," says Melis. "We just have to make better use of it."

\section{CARBON FIX}

The solar energy that plants capture is used to make chemical energy inside the cell. This is then used to fix $\mathrm{CO}_{2}$ and make sugars. This second stage, called the Calvin cycle, could also be made more efficient.

Rubisco is one of the most important enzymes in the Calvin cycle, but the $\mathrm{CO}_{2}$ fixing enzyme is prone to errors. "Every fourth turnover, it fixes an oxygen molecule instead of $\mathrm{CO}_{2}$," explains Donald Ort, a plant biologist for the US Department of Agriculture who is based at the University of Illinois at Urbana-Champaign. This chemical error, known as photorespiration, generates a toxic compound called glycolate that plants must break down.

When photosynthetic cells first evolved, there was very little oxygen in the atmosphere, so the fact that rubisco couldn't always distinguish oxygen from $\mathrm{CO}_{2}$ wasn't a problem. Researchers think that by the time oxygen levels were high enough to be a problem, the enzyme was too established and complex to be improved through evolution. Instead, plants make rubisco in large quantities so there is always enough to fix the $\mathrm{CO}_{2}$ needed. The enzyme is thought to be the most abundant on Earth.

Although higher levels of $\mathrm{CO}_{2}$ in the atmosphere boost photosynthesis, human activities that increase the level of $\mathrm{CO}_{2}$ will not improve rubisco's odds. The enzyme makes more mistakes at higher temperatures, and plant scientists expect that photorespiration will increase as a result of rising global temperatures - to the detriment of crop yields. 


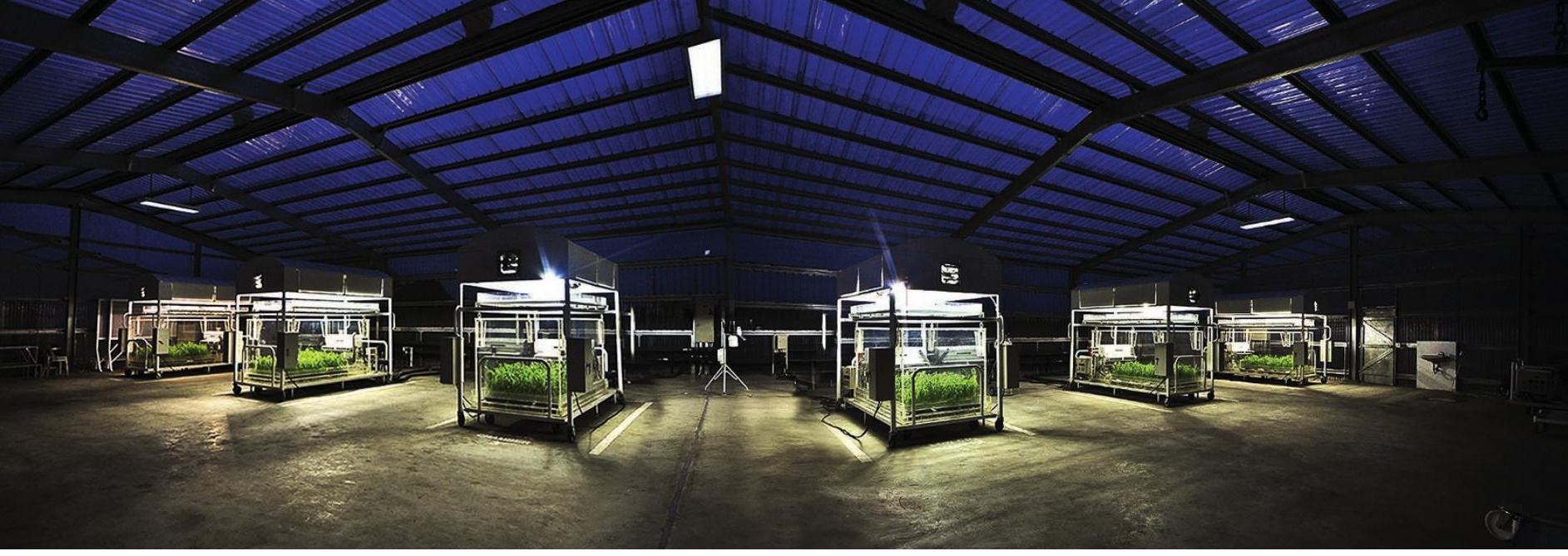

Plants grown in low $\mathrm{CO}_{2}$ chambers at the International Rice Research Institute in Los Baños, Philippines.

For many years, researchers were uncertain whether there was much variation in rubisco, says Elizabete Carmo-Silva, a plant scientist at Lancaster University, UK. But improvements in technology that allows photosynthetic phenotypes to be measured in the field have shown that this isn't true.

Carmo-Silva's group is studying rubisco and rubisco activase (which helps restore the carbon-fixing enzyme after it makes a mistake) in wheat strains. The work is ongoing, but the team has already found variants of these enzymes that recover more quickly than typical rubisco after fixing oxygen. These variants could be bred into the germplasm, the genetic material that's used to develop seeds, she says (see page S8).

Ort is focused on streamlining the reactions that plants use to break down glycolate. These type of reactions use a lot of energy and require chemical intermediates to be shuttled between chloroplasts, mitochondria and organelles called peroxisomes. "This lowers the efficiency of photosynthesis by about 30 or $40 \%$ - it's huge," says Ort.

Organisms, such as the bacterium Escherichia coli, have more-efficient reaction pathways for metabolizing glycolate than the complex pathways that evolved in plants. When researchers tried to introduce this bacterial mechanism into Arabidopsis, they found that photosynthesis was boosted only modestly ${ }^{3}$. Ort suspects that this is because plant cells were still shipping glycolate out of the chloroplast even with the introduction of the improved chemical pathway. Ort has developed tobacco plants with chloroplasts that lack glycolate transporters, and so are forced to metabolize the compound in that organelle using the more-efficient pathway. Preliminary results suggest that these plants had a $20-30 \%$ increase in biomass. Before submitting the work for publication, Ort plans to replicate the results in a second field experiment this year.

\section{REBUILDING RICE}

Although plants haven't developed an alternative to rubisco, a more-efficient form of photosynthesis has independently evolved more than 60 times. $\mathrm{C}_{4}$ photosynthesis, in which the first step involves producing a four-carbon compound instead of a three-carbon one, is about $50 \%$ more efficient than the $\mathrm{C}_{3}$ process because it uses a more-effective enzyme to capture $\mathrm{CO}_{2}$. This is especially true for plants living in dry, hot conditions, says Jane Langdale, a plant biologist at the University of Oxford, UK.

Langdale is part of the $\mathrm{C}_{4}$ Rice Project, which aims to transform rice from a $\mathrm{C}_{3}$ into $\mathrm{a}_{4}$ plant. The project was conceived in 1999 by John Sheehy, a plant physiologist at the International Rice Research Institute (IRRI) in Los Baños, Philippines, and the project's scientists would be the first to admit that their goal is ambitious. To make rice into $\mathrm{a}_{4}$ plant, researchers must endow it not only with new metabolic networks, but also with a new anatomy.

During the initial phases of the project, scientists focused on finding the genes responsible for $\mathrm{C}_{4}$ metabolism. They then added these genes to a strain of rice one at a time. In the modified rice, only about $5 \%$ of $\mathrm{CO}_{2}$ enters the moreefficient $\mathrm{C}_{4}$ pathway, says plant biologist Paul Quick at the IRRI, who led this phase of work. "We won't be able to get the benefit of the $\mathrm{C}_{4}$ pathway until we have the anatomy," he says. Understanding how to build the leaf anatomy is the current phase of the project.

Photosynthetic cells in the leaves of $\mathrm{C}_{4}$ plants are arranged around the veins in a circular pattern called Kranz anatomy (see 'Shape of things to come'). Instead of using rubisco, mesophyll cells use the enzyme phosphoenolpyruvate carboxylase, which doesn't bind oxygen, to capture $\mathrm{CO}_{2}$ in a four-carbon compound. The compound is then shuttled into a second type of cell, called a bundle sheath cell, where it is converted back to $\mathrm{CO}_{2}$ and fixed by rubisco. The $\mathrm{CO}_{2}$ concentration is much higher in $\mathrm{C}_{4}$ bundle sheath cells, and so the rate of photorespiration is much lower. Despite the additional con-version steps and the need to move compounds between cells, this process is more efficient than $\mathrm{C}_{3}$ photosynthesis.

To build Kranz anatomy into rice leaves, plant biologists need a developmental map. They don't know what sets a plant cell on the path to becoming part of a vein, a mesophyll or bundle sheath cell, and they don't know how these cells form the spatial patterns typical of $\mathrm{C}_{4}$ plants. Langdale has been studying development in maize, because it's one of only a few plants with both $\mathrm{C}_{3}$ leaves (the blades around the corn cob) and $\mathrm{C}_{4}$ leaves (all the rest). By examining the differences in gene-expression patterns between these leaf types, Langdale has found about 280 genes that seem to be involved in the formation of this Kranz anatomy. After whittling down the list to 70, based on function, her group "took each gene and put it into rice with a promoter to switch it on all the time", she explains. Not all of the genes seemed to have an effect. Langdale is now doing further studies of ten or so of the genes that seem the most promising.

Genetic engineering has advanced substantially since the $\mathrm{C}_{4}$ Rice Project started. Researchers used to have to transform rice one gene at a time, now they can do two or three genes at once. Eventually, they expect to be able to build a $\mathrm{C}_{4}$ shuttle - a set of genes required for $\mathrm{C}_{4}$ photosynthesis that can be inserted into various plants in one go. But first they must work out what changes to make. Langdale estimates that, realistically, $\mathrm{C}_{4}$ rice won't make an appearance in farmers' fields until at least 2039.

In the past, improving yields through photosynthetic interventions was not a practical research focus - these were "intellectual questions", says Christine Raines, a plant scientist at the University of Essex in Colchester, UK. Now the idea is in vogue, and researchers have the funding to test their ideas. "We will begin to see a lot of papers coming out in the next 18 months," she says.

But significant challenges lie ahead. "Organisms resist attempts to reprogram them," says Melis, and these projects seek to alter the very heart of a plant's chemistry. Before they can make such unprecedented changes, scientists will need, for example, to better understand how resources such as sugars and lipids are routed to certain places in plant cells.

Although this will be difficult, many plant biologists think that engineering photosynthesis could be the best hope for feeding a growing population and combating food insecurity. "Photosynthesis is the last big unexplored route to improving yields," says Harbinson.

Katherine Bourzac is a freelance journalist in San Francisco, California.

1. Kromdijk, J. et al. Science 354, 857-861 (2016).

2. Chen, M. et al. Science 329, 1318-1319 (2010)

3. Kebeish, R. et al. Nature Biotech. 25, 593-599 (2007). 